\title{
Extending the lifetime of resonant atmospheric particulate mass sensors with solvent rinses
}

\author{
Arthur T. Zielinski ${ }^{1 *}$, Nicole E. Weckman²*, Roderic L. Jones ${ }^{1}$, Markus Kalberer ${ }^{1}$, and Ashwin A. \\ Seshia ${ }^{2 * *}$ \\ ${ }^{1}$ Centre for Atmospheric Science, Department of Chemistry, University of Cambridge, CB2 1EW, Cambridge UK \\ ${ }^{2}$ The Nanoscience Centre, Department of Engineering, University of Cambridge, CB3 OFF, Cambridge UK \\ * Student Member, IEEE \\ ** Senior Member, IEEE
}

Received 1 Nov 2016, revised 25 Nov 2016, accepted 30 Nov 2016, published 5 Dec 2016, current version 15 Dec 2016.

\begin{abstract}
The cleaning of a collection-based sensor extends its lifetime and reduces its effective cost. Existing cleaning regimes for silicon-based devices typically require access to large laboratory equipment. A simple cleaning method based on solvent rinses is presented here for the application of microresonator atmospheric particulate mass sensors. The suggested approach is intended for scenarios with limited access to laboratory equipment. Two piezoelectric resonator topologies (in-plane bulk mode and out-of-plane flexural) collected particles via impaction for an hour before rinsing. The solvent rinses reset the resonant frequency and quality factor of each resonator to within $0.4 \%$ and $10 \%$ of their original values, respectively. Subsequent mass collections were largely repeatable despite fluctuations in particle concentration and deposition location. The presented method provides a straightforward but effective cleaning method for soluble particulate removal. A physical cleaning method is required after substantial insoluble particle adsorption.
\end{abstract}

Index Terms-flexural, LBAR, mass sensing, particulate matter.

\section{INTRODUCTION}

A wide range of sensing applications have emerged for microelectromechanical system (MEMS) resonators including temperature [1], pressure [2], and particulate mass sensing [3]-[5]. Some applications, such as mass sensing, require the adsorption of material onto the resonator surface which inherently limits the lifetime of the sensor. In principle if such a device is mass produced at a low cost then it can simply be replaced but practically this is rarely the case. Resonators may be fully integrated into the instrument making replacement difficult and new resonators, even of the same design, may require initial characterization. The other approach is to remove the adsorbed material with a cleaning protocol. The cleaning of silicon surfaces can be performed using a range of methods including $\mathrm{CO}_{2}$ snow jet [6]; ultraviolet radiation and ozone [7]; plasma cleaning [8]; and solvent soaks [7], [9], [10]. Traditional cleaning methods, such as ultrasonic baths [5], have also been applied to MEMS particulate mass sensors. These methods, although effective, are limited in their out-of-laboratory applicability where constraints exist on equipment size and materials. In the push from laboratory to widespread use, resonant MEMS sensors require a straightforward cleaning approach.

By extending microfluidic solvent applications outside of a flow cell [9], [10], a new rinsing technique is applied to resonant mass sensors whereby small volumes of common solvents are used to remove adsorbed particles without damaging wire bond connections. The technique is fast (less than five minutes) and can be applied in non-laboratory settings as it only requires hand-held equipment. The approach is inherently limited by the presence of insoluble particulates but is still relevant given that a significant portion of atmospheric particles are soluble in either water or methanol (with the exact percentage depending on the specific time and location) [11]. The simple solvent method therefore extends the lifetime until a traditional physical removal method is required.

This work investigates the effectiveness of the rinsing technique in terms of the reusability of an in-plane bulk acoustic wave resonator and a plate resonator operating in a flexural mode. The cleaning procedure was tested with two atmospherically relevant particle types and repeated three times for the same resonator. In all cases the resonant frequency returned to within $0.4 \%$ of the original value.

\section{METHODS}

For each resonator-particle combination, particles were collected for one hour followed by the cleaning of the resonator surface. This process was repeated three times for each combination. Optical images of the resonator surfaces were acquired with a digital microscope (VHX-500F, Keyence) before and after each cleaning step to qualitatively assess the cleaning. Throughout particle collection the resonant frequency of the resonator was tracked as it shifted negatively following the standard mass loading relationship [12]. The production and collection of particles onto the resonator follows the experimental procedure described earlier using the MEMS Impactor Stage (MIS) [13]. The arrangement allows for a correlation between the measured frequency shift of the resonator and 
the particle concentration of the incoming flow to be obtained. The following subsections highlight experimental details.

\section{A. Description of resonators and signal processing}

Both resonators, shown in Fig. 1, are fabricated using a commercial foundry process [14] starting from silicon-on-insulator wafers with aluminum nitride as the piezoelectric film. The in-plane bulk acoustic wave resonator, previously described in detail by Prasad et. al [12], is a suspended $1400 \mu \mathrm{m} \times 1400 \mu \mathrm{m}$ plate with four triangular electrodes. Its $\sim 3.15 \mathrm{MHz}$ square-extensional mode was tracked for the purpose of this study. The nearby wine-glass $(\sim 2.84 \mathrm{MHz})$ and butterfly $(\sim 3.20 \mathrm{MHz})$ modes were electrically suppressed. The flexural mode plate resonator, previously described in detail by Weckman and Seshia [15], is an $880 \mu \mathrm{m} \times 860 \mu \mathrm{m}$ plate with two rectangular electrodes. Its $\sim 350 \mathrm{kHz}(1,2)$ square plate flexural mode was tracked for this study. Resonators were transduced in a two-port configuration with actuation and sensing from two separate sets of electrodes.

The frequency response of each resonator was measured in an open-loop arrangement using a network analyzer (N9915A, Agilent) each minute with discrete frequency resolutions of $\pm 25 \mathrm{~Hz}$ and $\pm 12.5 \mathrm{~Hz}$ for secondary organic aerosol and salt collection, respectively. The in-plane resonator was driven at a $-10 \mathrm{dBm}$ while the flexural mode was driven at $0 \mathrm{dBm}$.
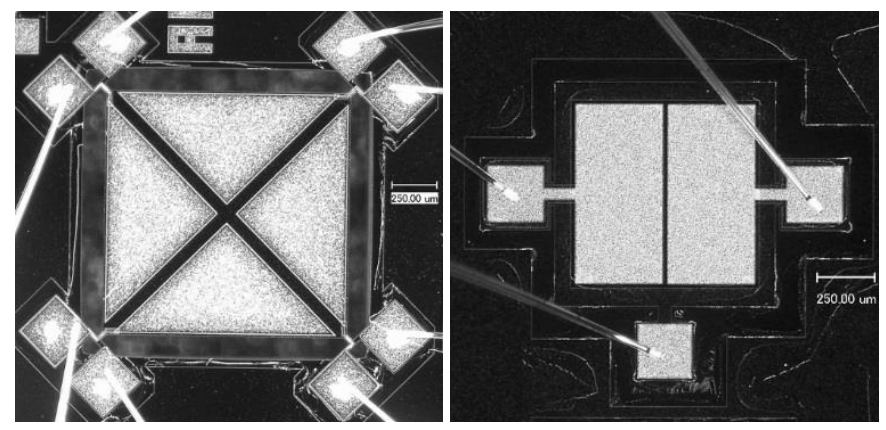

Fig. 1. Optical images of front resonator surfaces at 100x magnification. The in-plane (left) and flexural mode (right) resonators have lateral dimensions of $1400 \mu \mathrm{m} \times 1400 \mu \mathrm{m}$ and $880 \mu \mathrm{m} \times 860 \mu \mathrm{m}$, respectively. Particles collected on back surface.

\section{B. Particle production and collection}

The two particles used were secondary organic aerosol (SOA) from the ozonolysis of $\alpha$-pinene and ammonium sulfate, $\left(\mathrm{NH}_{4}\right)_{2} \mathrm{SO}_{4}$, salt particles produced through nebulization. SOA and ammonium sulfate significantly contribute to atmospheric particle compositions from both anthropogenic and natural sources [16]. Ozonolysis of $\alpha$-pinene (98\%, Sigma-Aldrich) involves flowing synthetic air (Zero grade, BOC) through an ozone generating lamp (3SC-9, UVP) with a primary emission wavelength of $184.9 \mathrm{~nm}$. The resulting flow of ozone was mixed with a flow of volatile $\alpha$-pinene in a $5 \mathrm{~L}$ flow tube at flow rates of $0.2 \mathrm{LPM}$ and 1.0 LPM, respectively. Ozonolysis reaction products subsequently condense to form organic particles mimicking atmospheric particle formation processes. Any remaining volatile compounds were removed by a charcoal denuder before size selection. The salt particles ( $\geq 99.0 \%$ Ammonium sulfate, SigmaAldrich) are nebulized with dry nitrogen gas (oxygen-free, BOC) from a $15 \mathrm{mM}$ solution in water (Optima LC/MS, Fisher Chemical) and dried through a diffusion dryer before size selection.
Once particles are produced they are electrostatically size selected at $\sim 300 \mathrm{~nm}$ using a differential mobility analyzer (DMA, Model 3081, TSI). Particles are then collected onto the resonator by inertial impaction using the MIS through a nozzle with $3 \times 0.25 \mathrm{~mm}$ circular jets. The cut-off diameter (the diameter at which $50 \%$ of particles are collected) is estimated to be $<200 \mathrm{~nm}$ based on empirical pressure drops and established analytical relationships for the given flow rate [17], [18]. Reference particle concentrations are measured with two condensation particle counters (CPCs) upstream and downstream of the MIS (Models 3775 and 3776, TSI). Flow through the setup was driven by a vacuum pump which kept the flow through the MIS at $\sim 0.7$ LPM producing a pressure drop on the order of $\sim 35 \mathrm{kPa}$.

Particles were collected for an hour during each experimental run. Prior to collection, particle production was allowed to stabilize for up to 40 minutes (e.g. to allow the ozone generating lamp to warm up) before flushing the system and pressurizing the MIS with clean flow.

\section{Cleaning procedure}

The removal of adsorbed particulate matter from the resonator surface was accomplished using $10 \mu \mathrm{L}$ solvent rinses deposited via a micropipette onto resonators held at angles of $45-60^{\circ}$ from horizontal to aid liquid drainage. Rinses were performed in ten second intervals before using a drying flow of nitrogen (4 LPM across the sensor) to expedite solvent evaporation. Resonators, which were cleaned prior to the initial collection, remained bonded to their chip carriers throughout. Cleaning conducted at room temperature $\left(20^{\circ} \mathrm{C}\right)$ and resonators inserted into MIS within 15 minutes of removal.

For the removal of SOA, three rinses with methanol (HPLC grade, Fisher Chemical) were applied before drying. For the removal of salt, the primary solvent was water (Optima LC/MS, Fisher Chemical) in conjunction with methanol (a more volatile solvent). The procedure alternated between methanol and water with five methanol rinses and three water rinses before drying with nitrogen. Methanol rinses began and concluded the procedure to improve resonator wetting and to limit the formation of "coffee-ring" stains [19] on the surface, respectively. The method for removing salt is also applicable for a mixture of salt and SOA, although such a mixture was not studied here.

In situations where significant soluble contaminants visibly persisted, probably due to ineffective solvent removal, the procedure was repeated. Insoluble contaminants from the solvents were always present during cleaning and accumulated after each rinse. Although assumed to be negligible, they can be removed after significant build-up using the physical processes described in the introduction.

\section{RESULTS AND DISCUSSION}

The reusability of each resonator is a combination of how well they can be restored to their original state and how well they can replicate the same measurement after cleaning. The restoration of a resonator is primarily a measure of the cleaning effectiveness while the ability to replicate measurements is also influenced by the flow setup.

Representative images of the in-plane resonator before and after cleaning are shown in Fig. 2 for each particle type. They show a clear removal of most particles where the remaining contaminants are insoluble in water and/or methanol. The cleaning effectiveness is corroborated by the recovery of both the resonant frequency and 
quality factor after each run as summarized in Table 1. The clean (i.e. 'before') resonant frequency of the in-plane resonator fell within a $275 \mathrm{~Hz}$ (equivalent to $87 \mathrm{ppm}$ ) and $412 \mathrm{~Hz}(131 \mathrm{ppm})$ span for SOA and salt collection, respectively. The total span of the six in-plane runs, performed with the same resonator, is $537 \mathrm{~Hz}(170 \mathrm{ppm})$ despite minor contamination (as seen in Fig. 2) between tests. Similarly, the quality factor remained within $10 \%$ of the original value after each cleaning across the six runs. The flexural resonator had a clean frequency span of $1387 \mathrm{~Hz}$ (equivalent to $0.39 \%$ ) with the frequency lowering with each run, but quality factors were more reliable returning to within $5 \%$ of the original value. Although the cleaning is less effective than the nearly full regeneration of ultrasonic cleaning [5], the resonant frequencies are still largely reset.
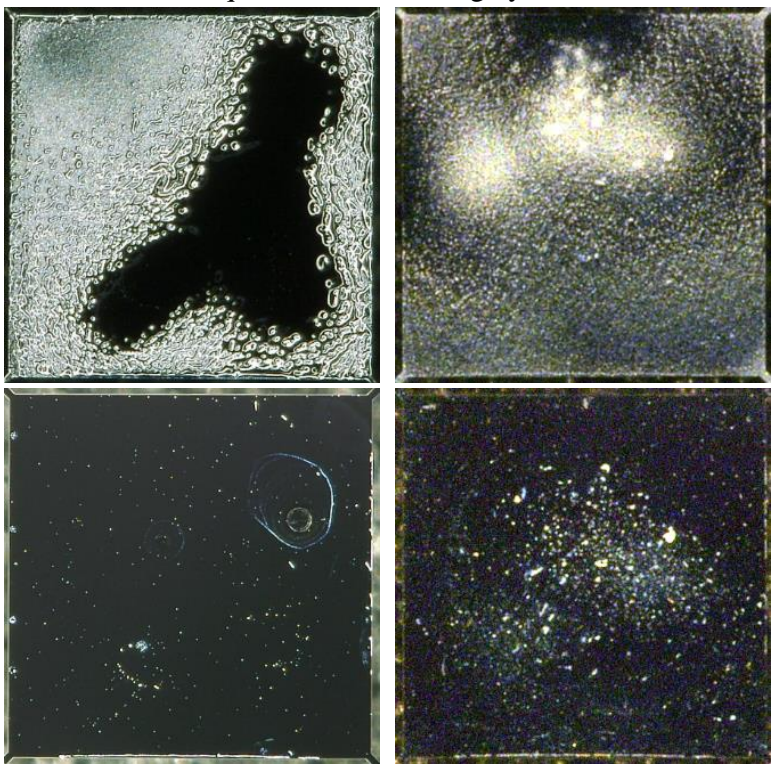

Fig. 2. Collection surface of in-plane resonator before (top) and after (bottom) cleaning of SOA (left) and salt (right). Images taken between first and second runs. Some particles remain (due to being insoluble in given solvents) but cleaning is predominantly effective. Contaminants in cleaned salt image (bottom right) were present at start of salt tests. For reference, the resonator side length is $1400 \mu \mathrm{m}$.

The frequency variation can be explained in part by the accumulation of contaminants (insoluble in methanol) on the resonator surface with each subsequent collection. The other major source of frequency variation is temperature as values in Table 1 are not corrected for temperature differences. Temperatures were relatively stable (within $1{ }^{\circ} \mathrm{C}$ ) for the in-plane resonator between runs of the same particle type, but the temperature between particle types varied by approximately $6{ }^{\circ} \mathrm{C}$. Using the temperature correction factor of $-102 \mathrm{~Hz} /{ }^{\circ} \mathrm{C}$ [12], the overall in-plane frequency span increases to $1150 \mathrm{~Hz}(365 \mathrm{ppm})$. In contrast, the flexural resonator experienced a temperature range of $5{ }^{\circ} \mathrm{C}$ between the three runs and has a higher temperature sensitivity of $-161 \mathrm{~Hz} /{ }^{\circ} \mathrm{C}$ (determined over a range of $22{ }^{\circ} \mathrm{C}$ to $57^{\circ} \mathrm{C}$ ). Accounting for temperature, the flexural frequency span reduces to $645 \mathrm{~Hz}(0.18 \%)$ which is $47 \%$ of the original variation.

Another minor issue for both resonators is the short time between cleaning and collection which may influence the measured clean frequency as the solvent(s) may not have fully evaporated. Typically, once the MIS is pressurized with clean air the resonant frequencies converge (e.g. the flexural span reduces to roughly $1000 \mathrm{~Hz}$ without temperature correction). There was not a significant performance difference between cleaning procedures, although in practice the inclusion of water rinses for salt removal increases the cleaning time for both application and evaporation. A potential improvement to the system would be to use higher grade solvents to avoid contamination during cleaning which was especially evident with methanol rinses. This would minimise contamination of other devices on the same chip which experience the "coffee-ring" effect when not dried effectively.

Table 1. Summary of changes in resonant frequency and quality factor before and after particle collection. Frequencies taken with MIS depressurized. Final clean results not included in table.

\begin{tabular}{|c|c|c|c|c|c|}
\hline & \multirow[b]{2}{*}{$\begin{array}{c}\text { Particle } \\
\text { (Run No.) }\end{array}$} & \multicolumn{2}{|c|}{ Resonant frequency $(\mathrm{Hz})$} & \multicolumn{2}{|c|}{ Quality factor } \\
\hline & & Before & After & Before & After \\
\hline \multirow{6}{*}{ 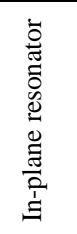 } & SOA (1) & $3,156,500$ & $2,978,850$ & 1,403 & 518 \\
\hline & SOA (2) & $3,156,225$ & $2,983,925$ & 1,403 & 485 \\
\hline & SOA (3) & $3,156,284$ & $2,971,759$ & 1,503 & 517 \\
\hline & Salt (1) & $3,155,963$ & $3,153,988$ & 1,365 & 1,342 \\
\hline & Salt (2) & $3,156,300$ & $3,152,913$ & 1,256 & 1,162 \\
\hline & Salt (3) & $3,156,375$ & $3,153,613$ & 1,343 & 1,261 \\
\hline \multirow{3}{*}{ 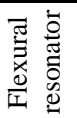 } & SOA (1) & 353,125 & 348,275 & 115 & 69 \\
\hline & SOA (2) & 352,800 & 344,313 & 111 & 74 \\
\hline & SOA (3) & 351,738 & 341,963 & 120 & 70 \\
\hline
\end{tabular}

The magnitude of the frequency response before (darker line) and after (lighter) particle collection of each resonator is shown in Fig. 3. Gray lines denote SOA collection while orange lines denote salt collection for the in-plane resonator. The in-plane resonator had a linear response with time as expected for a roughly constant particle concentration [3]. The flexural mode resonator began with a linear response which plateaued as the resonator approached saturation. Salt collection with the flexural mode resonator is not included due to the resonator's low mass sensitivity (on the order of 10 times lower than the in-plane resonator based on SOA collection).

Each resonator-particle pairing had similar particle collections, but some variation is evident. The cause is likely a combination of particle production instability and resonator spatial sensitivity.

Particle production of SOA is influenced by the volume of $\alpha$-pinene and the temperature of the ozone lamp. Additionally, the flow rate through the MIS can vary due to the nozzle clogging and vacuum pump fluctuations. These fluctuations are typically minor as compared to the total concentration of particles passing through the DMA which are on the order of 80,000 particles $/ \mathrm{cm}^{3}$. Minor fluctuations for salt production via nebulization are more significant due to the lower concentration of particles $\left(2,000\right.$ particles $\left./ \mathrm{cm}^{3}\right)$.

The spatial sensitivity of a resonator is based on the observed mode shape. If mass is collected near an anti-node the frequency response is maximized whereas collection near a node yields a negligible response [19]. The deposition pattern on the resonator will therefore influence the resonator response. Between runs the deposition location varied due to the slight variations (up to $\sim 200 \mu \mathrm{m}$ ) when replacing both the chip carrier and the lid of the MIS. As such the sensitivities of the resonator response are also expected to vary.

It is difficult to make substantial frequency comparisons between particle types due to markedly different concentrations. As a system, 
however, the MIS collection efficiency (i.e. the percentage of particles collected by the MIS as a whole) is consistent with previous studies [20] where $\alpha$-pinene SOA showed lower collection efficiencies than ammonium sulfate ( $30 \%$ vs $\sim 60 \%$ ) at low humidity.
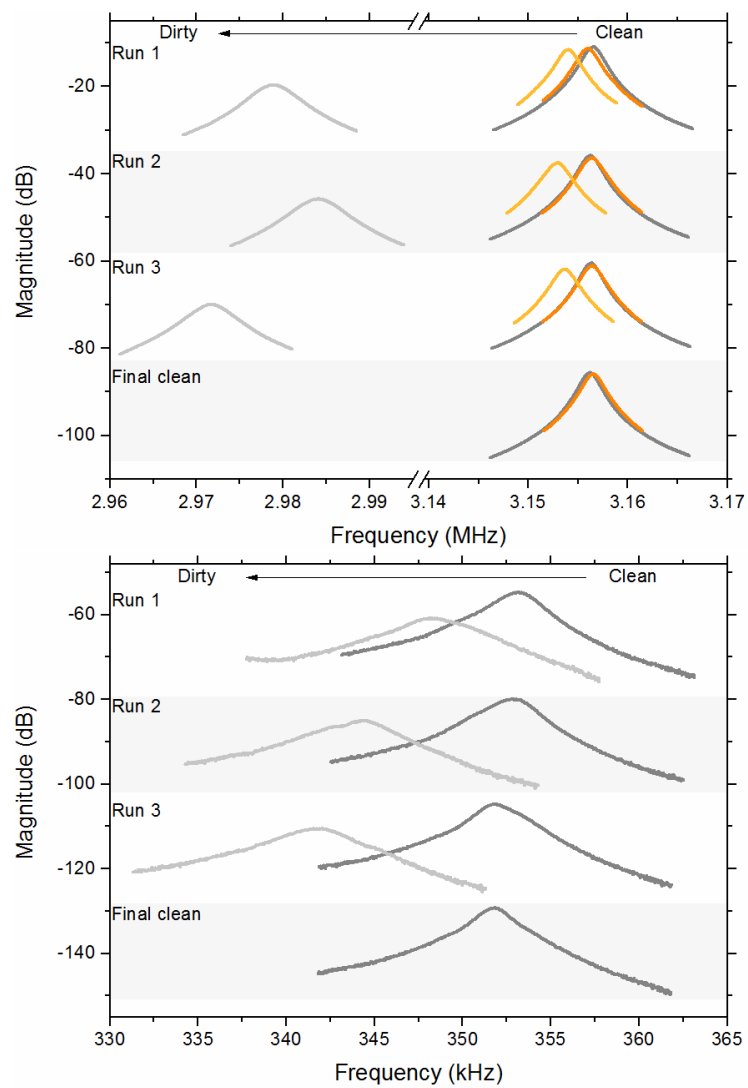

Fig. 3. Frequency response shifts due to one-hour particle collections on the in-plane (top) and flexural (bottom) sensors where the right-most, darker colored peaks are the cleaned frequency responses. SOA collection runs and salt (in-plane only) collection runs are depicted by grey and orange lines, respectively. Each row represents one experimental run where resonators were cleaned between runs. Data does not account for differences in particle concentrations. Magnitude values are relative as each run is offset in the $y$-axis by $25 \mathrm{~dB}$.

\section{CONCLUSIONS}

A novel simple cleaning approach of MEMS resonators based on solvent rinses was introduced and studied using atmospherically relevant particles. Particles were collected by impaction onto the back face of both in-plane and flexural mode resonators. Solvent rinses were then used to reset the resonant frequency and quality factor of both resonators, returning them to within $0.4 \%$ and $10 \%$ of their original values over three repeats, respectively. Chips remained bonded to their chip-carriers throughout thus relinquishing the need for bulky, expensive equipment for resonator cleaning or re-bonding.

Although the method leaves a small amount of insoluble residue it provides sufficient cleaning to substantially extend the lifetime of resonator-based particle sensors before requiring more rigorous physical cleaning methods. The rinsing approach could therefore be included in an automated system to partially reset a particulate sensor on a regular interval extending the time between maintenance visits.

\section{REFERENCES}

[1] A. Cagliani, V. Pini, J. Tamayo, M. Calleja, and Z. J. Davis, "Ultrasensitive thermometer for atmospheric pressure operation based on a micromechanical resonator," Sensors Actuators, B Chem., vol. 202, pp. 339-345, 2014.

[2] K. E. B. Thornton, D. Uttamchandani, and B. Culshaw, "A sensitive optically excited resonator pressure sensor," Sensors Actuators A. Phys., vol. 24, no. 1, pp. 15-19, 1990.

[3] I. Paprotny, F. Doering, P. A. Solomon, R. M. White, and L. A. Gundel, "Microfabricated air-microfluidic sensor for personal monitoring of airborne particulate matter: Design, fabrication, and experimental results," Sensors Actuators A Phys., vol. 201, pp. 506-516, 2013.

[4] E. Mehdizadeh, V. S. Kumar, J. Wilson, and S. Pourkamali, "Inertial impaction on MEMS balance chips for real-time air quality monitoring," IEEE Sens. J., vol. 17, no. 8, pp. 2329-2337, 2017.

[5] H. S. Wasisto, S. Merzsch, E. Uhde, A. Waag, and E. Peiner, "Partially integrated cantilever-based airborne nanoparticle detector for continuous carbon aerosol mass concentration monitoring," J. Sensors Sens. Syst., vol. 4, no. 1, pp. 111$123,2015$.

[6] R. Sherman, "Carbon dioxide snow cleaning," Part. Sci. Technol., vol. 25, no. 1, pp. 37-57, 2007.

[7] M. P. Seah and S. J. Spencer, "Ultrathin $\mathrm{SiO} 2$ on Si. I. Quantifying and removing carbonaceous contamination," J. Vac. Sci. Technol. A Vacuum, Surfaces, Film., vol. 21 , no. 2 , p. $345,2003$.

[8] B. Anthony, "In situ cleaning of silicon substrate surfaces by remote plasmaexcited hydrogen," J. Vac. Sci. Technol. B Microelectron. Nanom. Struct., vol. 7 , no. 4 , p. 621,1989

[9] F. Lucklum, E. K. Reichel, and B. Jakoby, "Miniature density-viscosity measurement cell utilizing electrodynamic- acoustic resonator sensors," Sensors Actuators A Phys., vol. 172, no. 1, pp. 75-81, 2011.

[10] D. Sparks, R. Smith, R. Schneider, J. Cripe, S. Massoud-Ansari, A. Chimbayo, and N. Najafi, "A variable temperature, resonant density sensor made using an improved chip-level vacuum package," Sensors Actuators A Phys., vol. 107 no. 2, pp. 119-124, 2003.

[11] J. H. Seinfeld and S. N. Pandis, Atmospheric chemistry and physics: from air pollution to climate change, 2nd ed. Hoboken: John Wiley \& Sons, Inc., 2006

[12] A. Prasad, J. Charmet, and A. A. Seshia, "Simultaneous interrogation of high-Q modes in a piezoelectric-on-silicon micromechanical resonator," Sensors Actuators A Phys., vol. 238, pp. 207-214, 2016.

[13] A. T. Zielinski, M. Kalberer, R. L. Jones, A. Prasad, and A. A. Seshia, "Particulate mass sensing with piezoelectric bulk acoustic mode resonators," in Internationl Frequency Control Symposium (IFCS), 2016.

[14] MEMSCAP Inc., "PiezoMUMPs Design Handbook." 2014

[15] N. Weckman and A. Seshia, "Micromachined piezoelectric acoustic sensor with multiple addressable flexural modes demonstrating improved $\mathrm{Q}$ in liquid," in EUROSENSORS, 2015, vol. 120, pp. 1003-1006.

[16] IPCC, "Climate change 2013: The physical science basis. Contribution of working group I to the fifth assessment report of the intergovernmental panel on climate change." 2013.

[17] S. V. Hering, "Calibration of the QCM impactor for stratospheric sampling," Aerosol Sci. Technol., vol. 7, no. 3, pp. 257-274, 1987.

[18] R. E. Hillamo and E. I. Kauppinen, "On the performance of the Berner Low Pressure Impactor," Aerosol Sci. Technol., vol. 14, no. 1, pp. 33-47, 1991.

[19] A. T. Zielinski, A. Prasad, A. A. Seshia, M. Kalberer, and R. L. Jones, "Effects of spatial sensitivity on mass sensing with bulk acoustic mode resonators," Sensors Actuators A Phys., vol. 236, pp. 369-379, 2015.

[20] E. Saukko, A. T. Lambe, P. Massoli, T. Koop, J. P. Wright, D. R. Croasdale, D. A. Pedernera, T. B. Onasch, A. Laaksonen, P. Davidovits, D. R. Worsnop, and A. Virtanen, "Humidity-dependent phase state of SOA particles from biogenic and anthropogenic precursors," Atmos. Chem. Phys., vol. 12, no. 16, pp. 7517 7529, 2012. 